\title{
Comparison of cardiothoracic surgical training before and during the COVID-19 pandemic in the United Kingdom
}

Ahmed Mohamed Abdel Shafi, MBBS, BSc, Amir Majid Sheikh, MD, FRCS(C/Th), and Wael Ibrahim Awad, MD, FRCS(C/Th)

\section{ABSTRACT}

Background: Cardiothoracic training during the Coronavirus disease 2019 (COVID-19) pandemic witnessed a dramatic change in the United Kingdom, resulting in changes in surgical provisions, centralization of services, and reduced surgical case volume. The aim of this study was to assess the impact COVID-19 on surgical training and seek opinions regarding the future role of surgical simulation in cardiothoracic training.

Methods: A cross-sectional survey was designed and distributed to 200 cardiothoracic national and non-national trainees in the United Kingdom. The survey consisted of 31 questions in 4 sections: Demographics, Service Provision, Training, and Simulation Training.

Results: Eighty-three of the 200 trainees (41.5\%) completed the survey, including 44 of 129 national trainees (34.1\%) and 39 of 71 non-national trainees $(54.9 \%)$. Sixty-seven respondents $(80.7 \%)$ agreed that cardiothoracic training had been negatively impacted as a result of COVID-19 $(P<.001)$. In addition, $12 \%$ agreed that adequate resources were available for learning/practicing technical skills, $87 \%$ agreed that simulation had a role in cardiothoracic training, $81 \%$ recognized simulations is an important tool in improving their surgical skills, and $79.5 \%$ agreed that simulation should be used to meet the increasing need in training/education moving forward.

Conclusions: COVID-19 has had a significant impact on surgical training, with concerns that these effects could have further implications downstream. Simulation training has been underused thus far, and trainees face an uphill challenge to enhance their skills and technical abilities in the operating room. Simulation is recommended by trainees and may represent a solution to the challenges of safe and effective cardiothoracic surgical training. (JTCVS Open 2021;7:394-410)

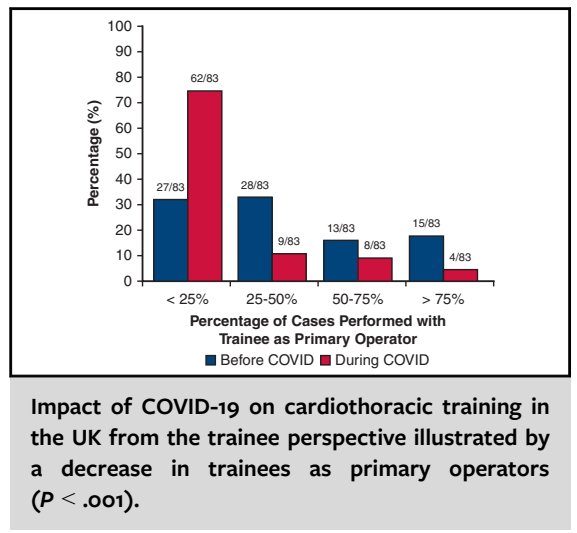

CENTRAL MESSAGE

COVID-19 has had a significant impact on surgical training, with potential future implications. Simulation offers a potential solution to the challenges faced as a result of COVID-19.

\section{PERSPECTIVE}

Surgical training across all disciplines have faced challenges as a result of COVID-19. We compare the effects of COVID-19 on cardiothoracic surgical training in the United Kingdom. Trainees are in agreement that simulation has a role in their training and an important tool in improving their surgical skills. We propose that all available resources be used to minimize the disruption to training

See Commentaries on pages 411 and 413 .
From the Department of Cardiothoracic Surgery, Barts Heart Centre, St Bartholomew's Hospital, London, United Kingdom.

Received for publication Oct 4, 2020; accepted for publication July 9, 2021; available ahead of print July 30, 2021.

Address for reprints: Wael Ibrahim Awad, MD, FRCS(C/Th), Department of Cardiothoracic Surgery, Barts Heart Centre, St Bartholomew's Hospital, West Smithfield, London EC1A 7BE, United Kingdom (E-mail: wael.awad@nhs.net).

2666-2736

Copyright (c) 2021 The Authors. Published by Elsevier Inc. on behalf of The American Association for Thoracic Surgery. This is an open access article under the CC BY-NCND license (http://creativecommons.org/licenses/by-nc-nd/4.0/).

https://doi.org/10.1016/j.xjon.2021.07.004 $\square$ Video clip is available online.

Coronavirus disease 2019 (COVID-19), caused by the novel severe acute respiratory syndrome coronavirus 2 , first appeared at the end of 2019, ${ }^{1}$ and was declared a pandemic by the World Health Organization on March 21, 2020. The COVID-19 pandemic has had an unprecedented impact 


\section{Abbreviations and Acronyms \\ CCT $=$ Certificate of Completion of Training \\ COVID-19 = Coronavirus disease 2019 \\ NTN = National Training Number \\ SCTS = Society for Cardiothoracic Surgery \\ ST $\quad=$ Specialty Trainee}

on global healthcare systems. The strain on healthcare resources despite best efforts has led to the need for rationing of medical equipment and interventions. The surgical workforce had had to adapt, and the Royal Colleges of Surgeons published guidelines that include fulfilling alternative surgical and nonsurgical roles. The emergency redeployment of surgical trainees to other sectors and frontlines has impacted not only the quantity, but also the quality of training as emphasis has moved to service provision.

Cardiothoracic services have been centralized and elective surgery has been curtailed in an effort to continue to provide emergency and urgent cardiac surgical care. ${ }^{2-4}$ As a result of the increased operative risks associated with COVID-19 infection, it has been agreed that only consultants or senior surgical trainees should operate on these higher-risk patients. Thus, in combination with the significant reduction in case volume, training opportunities have been greatly diminished.

On March 19, 2020, the Joint Committee on Surgical Training further announced that training rotations would be suspended to eliminate a possible additional burden on healthcare systems; consequently, trainees have remained within the same institution. Trainees needing more time to complete their training have been granted additional time to do so, to maintain the integrity of surgical training.

In addition, clinical and educational conferences/courses have been postponed in an effort to ensure that trainees are available to help healthcare services deal with the pandemic and minimize any potential risk of transmission. Although this measure has reduced any potential negative effects on the workforce and subsequently on patients, training has been further compromised.

Cardiothoracic training in the United Kingdom has undergone a dramatic change during the COVID-19 pandemic resulting from changes in surgical provision, centralization of services, reduced surgical case volume, and, subsequently, reduced training opportunities. The aims of the present study were to assess the impact of COVID-19 on surgical training and to explore the future role of surgical simulation in cardiothoracic training (see Video 1).

\section{METHODS}

\section{Survey Design and Distribution}

A retrospective online survey (www.forms.google.com) was developed to assess the impact of COVID-19 on cardiothoracic training and the role of

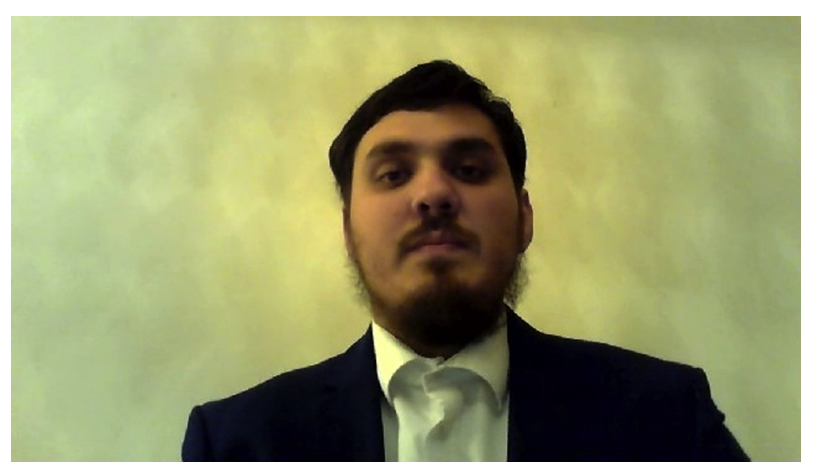

VIDEO 1. An overview of the study, the reasons behind it, and its methodology and results. Video available at: https://www.jtcvs.org/article/ S2666-2736(21)00181-9/fulltext.

simulation in cardiothoracic training among NTN (National Training Number; ie, designated) and non-NTN (ie, nondesignated) trainees in the United Kingdom. The survey's content was validated by a panel of 4 experts consisting of 2 senior cardiothoracic consultants (an educational supervisor and a training program director) involved in the education and training of both NTN trainees and non-NTN trainees and in the recruitment of non-NTN trainees to their institution, a medical educational manager who organizes and manages the educational needs of all trainees within our hospital, and an academic fellow previously involved in developing a national simulation curriculum (in urology) and in developing/validating surveys and curriculum designs in this field. It was further piloted among 5 trainees to ensure that the questions were easy to understand and appropriate before final dissemination.

The 31-question survey (Appendix 1) comprised 4 distinct sections: (1) demographics, (2) service provision, (3) training, and (4) simulation training in cardiothoracic surgery. Each question was critically analyzed to minimize the possibility of ambiguity and misinterpretation. This was distributed among national trainee representatives and educational managers at cardiothoracic centers through online invitations. Formal ethical approval was not required for this study. The survey was open from June 8, 2020, until July 10, 2020.

The survey focused on training before (January 1 to March 20, 2020) and during (March 21 to June 7, 2020) the COVID pandemic in the United Kingdom. An additional focus was to see whether trainees felt the need for further training to improve their technical skills and the possible role of simulation in this training. Participants who completed the survey did not receive any incentives.

\section{Statistical Analysis}

Data analysis was performed using SPSS 19.0 (IBM, Armonk, NY), and graphical illustrations were created with Excel (Microsoft, Redmond, Wash). A nonparametric $t$ test was performed to compare responses when deemed appropriate. A $P$ value $<.05$ was considered to indicate statistical significance.

\section{RESULTS}

\section{Demographics}

The survey was completed by 83 of 200 participants $(41.5 \%)$, including 44 of 129 NTN trainees $(34.1 \%)$ and 39 of 71 non-NTN trainees $(54.9 \%)$. The mean age of the trainees who completed the survey was 41 years ( 34.8 years for NTN trainees and 41 years for non-NTN trainees; $P<.001)$. The overall male:female distribution among participants was 72:11 (39:5 in NTN trainees and 33:6 in 
non-NTN trainees). Only 1 non-NTN trainee was not in full-time training. The average number of years in the specialty for non-NTN trainees was $4.57 \pm 3.81$. The NTN training pathway consists of 8 years, denoted ST1-ST8, with successful completion marked by a Certificate of Completion of Training (CCT). The NTN trainees included 14 of $44(31.8 \%)$ in their early years of specialty training $(<\mathrm{ST} 4), 14$ of $44(31.8 \%)$ in their intermediate years (ST5-ST6), 13 of $44(29.6 \%)$ in their later years (ST7-ST8), and 3 of $44(6.8 \%)$ post-CCT. The speciality and region of the participants are shown in Figure 1.

\section{Service Provision}

Before the COVID-19 pandemic, 59 of 83 trainees $(71.1 \%)$ were assigned to be residents on call; this number increased to 68 of $83(81.9 \%)$ during the pandemic $(P=.1)$. Thirty-eight of the 83 trainees $(45.8 \%)$ had a change in job role during the pandemic, with $25(30.1 \%)$ were redeployed to another specialty, including intensive care, general surgery, orthopedics, and medical specialties.

\section{Training}

The mean number of theatre sessions per week allocated to all trainees was 3.1 before the pandemic and decreased to 1.7 during the pandemic $(P<.001)$. There was no significant difference between NTN and non-NTN trainees in the mean number of weekly theatre sessions allocated before the pandemic (3.36 vs $2.82 ; P=.053$ ) and during the pandemic (1.84 vs $1.41 ; P=.104)$. However, both NTN and non-NTN trainees showed a significant difference from before the pandemic to during the pandemic (NTN, from 3.36 to $1.84[P<.0001]$; non-NTN from 2.82 to $1.41[P<.0001])$. Trainees also showed a drop in the number of cases in which they were the primary operator $(P<.001)$ (Figure 2). Training experience also changed in terms of training provided by the surgical trainer and time allocated to develop technical skills (Figure 3). The allocated time in theatre was deemed insufficient to gain the required technical skills by non-NTN trainees versus NTN trainees before the pandemic $(P=.0332)$ but not during the pandemic $(P=.6495)$. There was no difference between trainees attending theatres when not scheduled to gain more operative experience before $(P=.1874)$ or during $(P=.8883)$ the pandemic, but there was a difference in the perceived level of training provided by non-NTN versus NTN trainees before the pandemic $(P=.0089)$, but not during the pandemic $(P=.2717)$. Training supplementation is shown in Figure 4. Twenty-eight of 83 trainees (33.7\%) felt that they would still be able to earn a CCT by the planned date.

\section{Simulation in Training}

In the year preceding COVID-19, 64 of 83 trainees $(77.1 \%)$ engaged in wetlab surgical simulation, 38
$(45.8 \%)$ with benchtop models, $11(13.3 \%)$ with virtual reality models, with $12(14.5 \%)$ engaging with no simulation. As a consequence of COVID-19, trainees were asked how future training/education needs could be met using different training modalities (Figure 5). There was no difference in the mean Likert score between NTN and non-NTN trainees in any of the training modalities: simulated surgical procedures, 3.77 versus 4.13 $(P=.125)$; E-learning, 3.45 versus $3.79(P=.153)$; or webinars, 3.66 versus $3.82(P=.518)$. The majority of trainees agreed or strongly agreed that simulation training has a role in cardiothoracic surgery, and that a series of remote wetlab sessions would be useful $(87 \%$ and $82 \%$, respectively) (Figure 6). The procedures that trainees would find most useful in cardiothoracic surgery are shown in Figure 7.

Suggestions by trainees, using an open-ended question, on how future training/educational needs could be met included cross-cover online teaching between different centers, including cardiology, personalized mentorship, and funding for departments to purchase simulation equipment (eg, pig hearts), to allow trainees to continue to develop their skills in light of the reduced operating opportunities.

\section{DISCUSSION}

Cardiothoracic surgeons in the United Kingdom currently train for at least 10 years after achieving a primary medical qualification. Traditionally, training in cardiothoracic surgery commences at the ST3 level after completion of 2 years of core surgical training preceded by 2 years of foundation training following general medical qualification. Specialty training runs from ST3 to ST8, at which point an exit exam is needed. However, since 2013 recruitment, also has been directly at the ST1 level with training running from ST1 to ST8. Trainees initially achieve competence in general surgery and the range of cardiothoracic practices and subsequently hone their training in the latter years to adult cardiac surgery or general thoracic surgery. Subspecialist practice commonly requires further exposure through fellowships, including congenital cardiothoracic surgery and transplant surgery. ${ }^{5}$

Currently, 129 NTN and 71 non-NTN trainees in the UK registered with the Society of Cardiothoracic Surgeons (SCTS). Cardiothoracic trainees are expected to have performed a minimum of 250 major cases by the time of final certification. ${ }^{6}$ NTN trainees are allocated to General Medical Council-approved training posts providing a structure for meeting these requirements and earn a CCT. However, non-NTN trainees are employed at institutional levels with no set criteria for employment, mainly for service provision with limited allocated training. They must provide evidence that they have achieved some or all of the required 


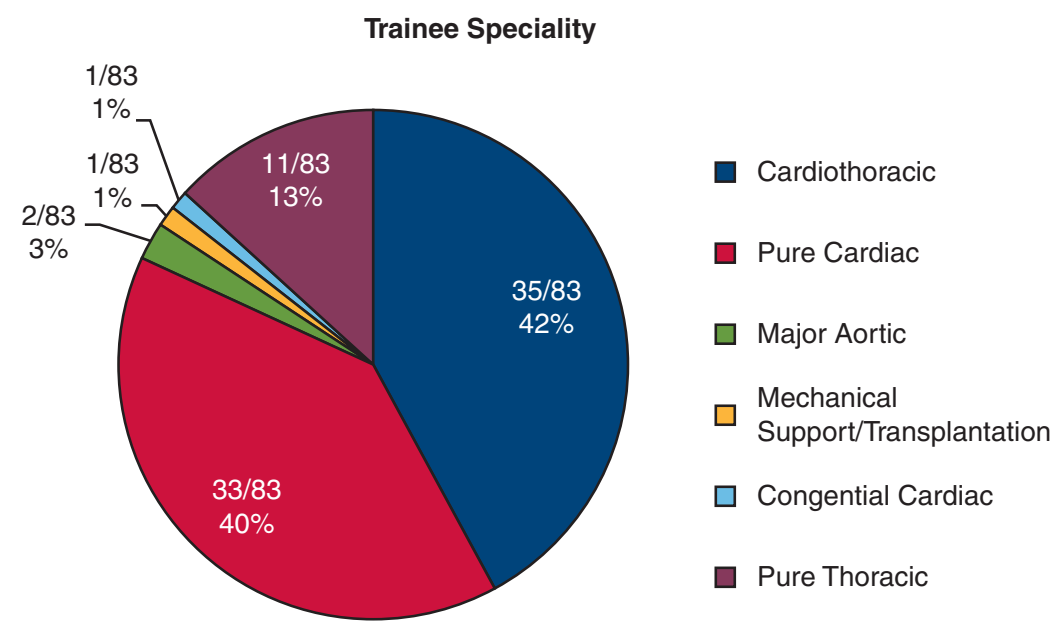

A

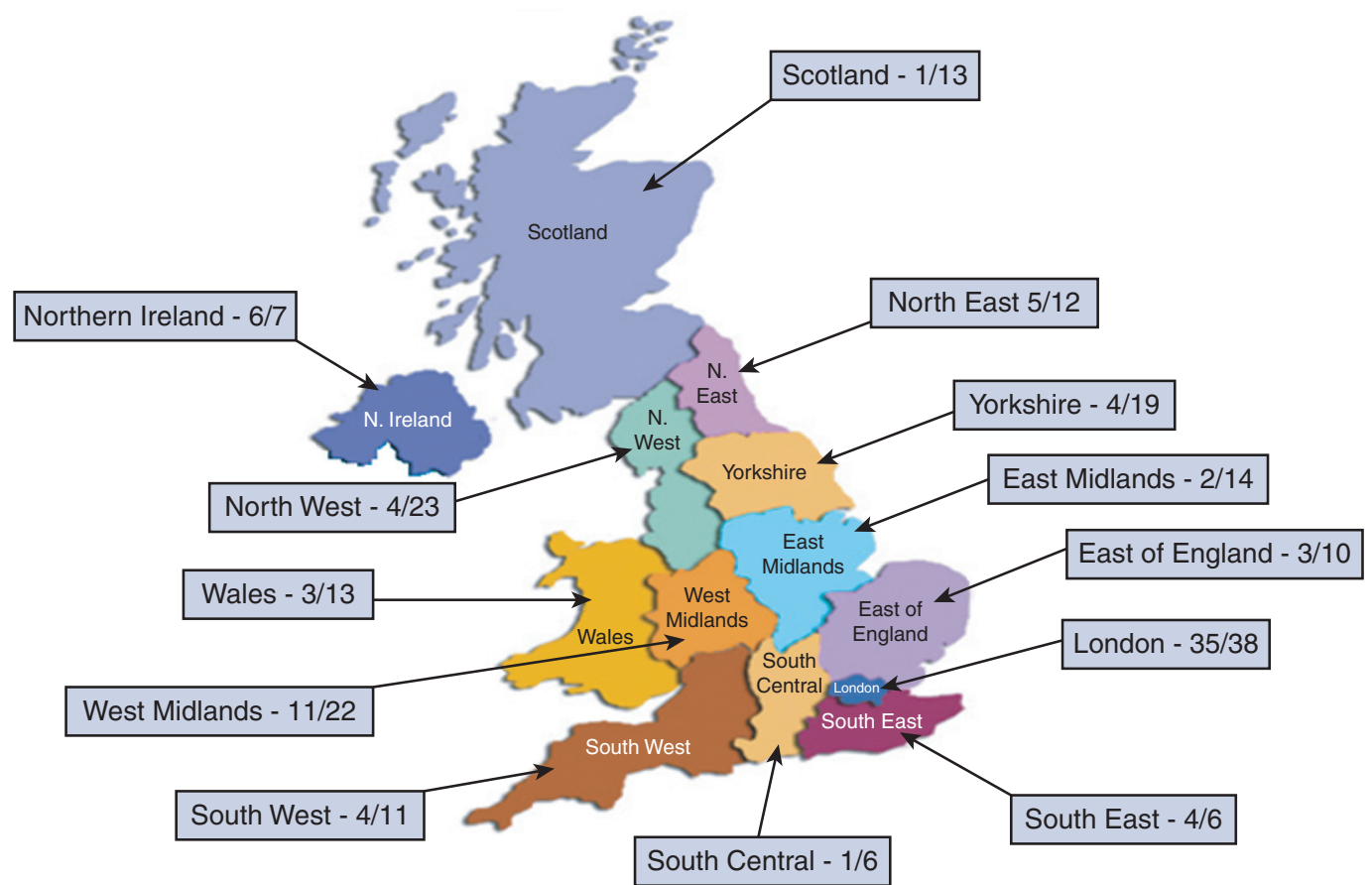

\begin{tabular}{|c|c|c|c|c|c|c|c|c|c|c|c|c|c|c|}
\hline & Scotland & $\begin{array}{c}\text { North } \\
\text { East }\end{array}$ & Yorkshire & $\begin{array}{c}\text { East } \\
\text { Midlands }\end{array}$ & $\begin{array}{l}\text { East of } \\
\text { England }\end{array}$ & London & $\begin{array}{l}\text { South } \\
\text { East }\end{array}$ & $\begin{array}{l}\text { South } \\
\text { Central }\end{array}$ & $\begin{array}{l}\text { South } \\
\text { West }\end{array}$ & $\begin{array}{c}\text { West } \\
\text { Midlands }\end{array}$ & Wales & $\begin{array}{l}\text { North } \\
\text { West }\end{array}$ & $\begin{array}{l}\text { Northern } \\
\text { Ireland }\end{array}$ & TOTAL \\
\hline NTN & $1 / 9$ & $3 / 10$ & $3 / 10$ & $1 / 11$ & $2 / 14$ & $15 / 18$ & $1 / 3$ & $0 / 4$ & $4 / 8$ & $6 / 14$ & $3 / 9$ & $2 / 15$ & $3 / 4$ & $44 / 129$ \\
\hline Non-NTN & $0 / 4$ & $2 / 2$ & $1 / 9$ & $1 / 3$ & $1 / 2$ & $20 / 20$ & $3 / 3$ & $1 / 2$ & $0 / 3$ & $5 / 8$ & $0 / 4$ & $2 / 8$ & $3 / 3$ & $39 / 71$ \\
\hline Total & $1 / 13$ & $5 / 12$ & $4 / 19$ & $2 / 14$ & $3 / 16$ & $35 / 38$ & $4 / 6$ & $1 / 6$ & $4 / 11$ & $11 / 22$ & $3 / 13$ & $4 / 23$ & $6 / 7$ & $83 / 200$ \\
\hline
\end{tabular}

B

FIGURE 1. A, Pie chart displaying the specialties of all trainees (National Training Number $[N T N]$ and non-NTN) currently in post. B, Geographical distribution of responding trainees (both NTN and non-NTN). The numbers in brackets are the numbers of responding trainees compared with the total number within that region, with the breakdown shown in the table.

competencies in nonapproved posts to be awarded an equivalent certification via the Certificate of Eligibility for Specialist Registration pathway. ${ }^{7}$

Doctors at all stages of training were affected by the COVID-19 pandemic and had their clinical routines disrupted, either by reduced training opportunities or by redeployment to other specialties. ${ }^{8,9}$ However, COVID-19 has had global ramifications across all specialties with the need for trainees to be redeployed to other specialties unavoidable and necessary to ensure that adequate care is 


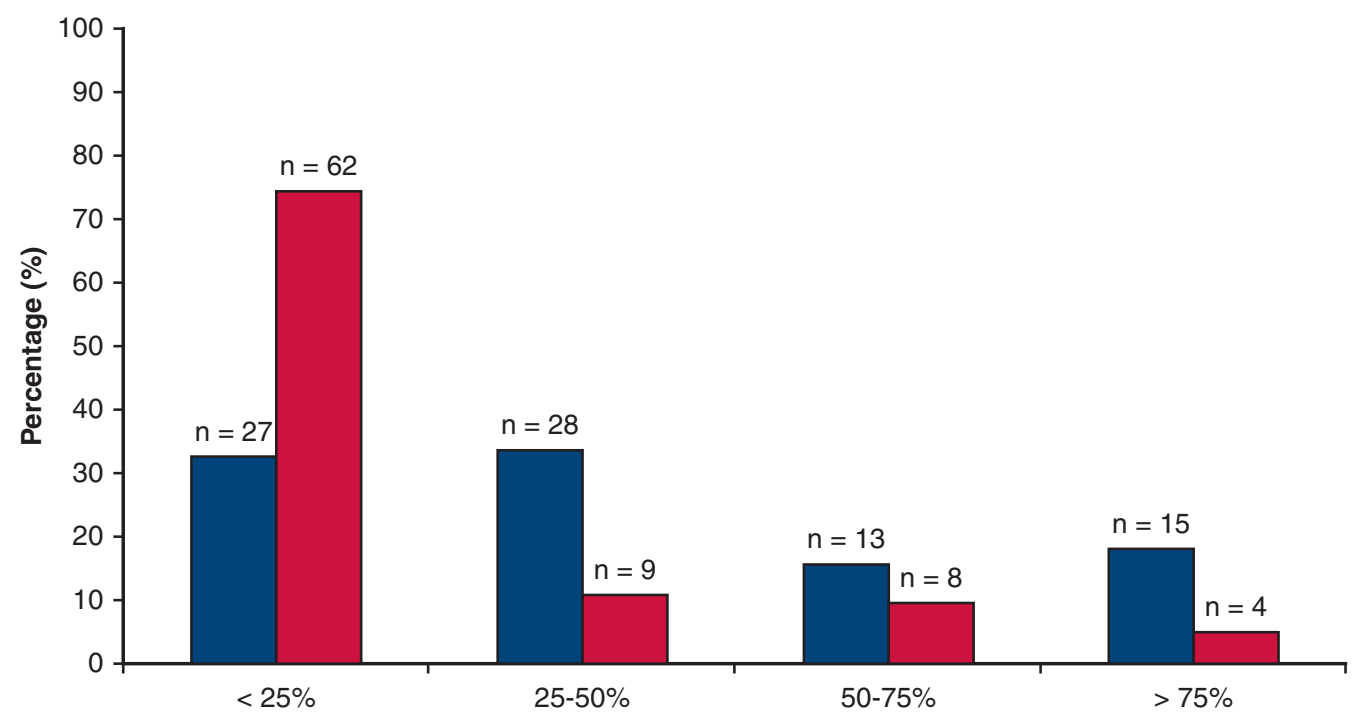

Percentage of Cases Performed with Trainee (/83) as Primary Operator

$\square$ Before COVID $\square$ During COVID

Before COVID

During COVID

\begin{tabular}{|l|c|c|c|}
\hline & NTN & Non-NTN & Total \\
\hline$<25 \%$ of cases & 11 & 16 & $\mathbf{2 7}$ \\
\hline $25-50 \%$ of cases & 16 & 12 & $\mathbf{2 8}$ \\
\hline $50-75 \%$ of cases & 7 & 6 & $\mathbf{1 3}$ \\
\hline$>75 \%$ of cases & 10 & 5 & $\mathbf{1 5}$ \\
\hline Total & $\mathbf{4 4}$ & $\mathbf{3 9}$ & $\mathbf{8 3}$ \\
\hline
\end{tabular}

\begin{tabular}{|l|c|c|c|}
\hline & NTN & Non-NTN & Total \\
\hline$<25 \%$ of cases & 30 & 32 & 62 \\
\hline $25-50 \%$ of cases & 6 & 3 & 9 \\
\hline $50-75 \%$ of cases & 4 & 4 & $\mathbf{8}$ \\
\hline$>75 \%$ of cases & 4 & 0 & 4 \\
\hline Total & $\mathbf{4 4}$ & 39 & $\mathbf{8 3}$ \\
\hline
\end{tabular}

FIGURE 2. Bar chart illustrating the impact of COVID-19 on trainees as primary operators, comparing the time periods before the pandemic (January 1 to March 20, 2020) and during the pandemic (Mar 21, 2020 to June 7, 2020) $(P<.001)$. NTN, National Training Number.

provided in the specialties in which the effect of COVID-19 was greatest. The impact of this on training perhaps may be even more significant in surgical disciplines. This is because surgery is a craft that entails consistent hands-on practice to develop and maintain the necessary technical skills.

The allocated time in theatre was sufficient to gain the required technical skills

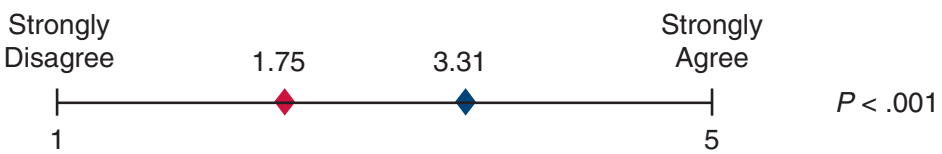

I attended theatres, when not scheduled to do so, to gain more operative experience

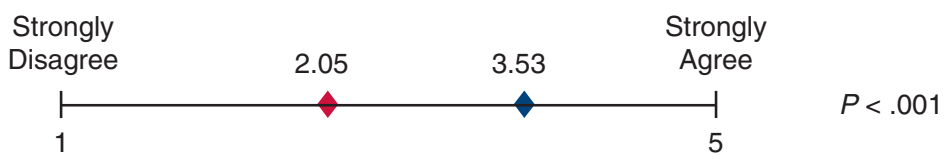

My surgical trainer was able to provide me with the training I required during this period

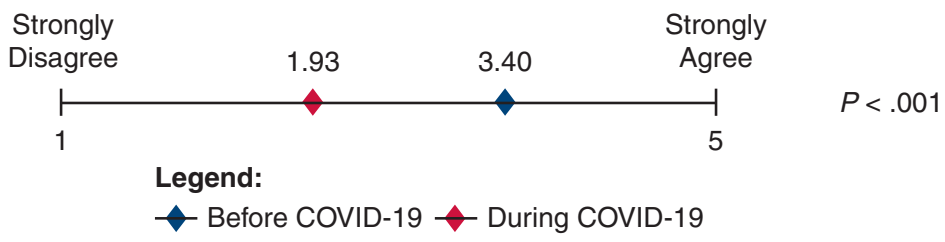

FIGURE 3. Scale comparison using the mean Likert scores $(1=$ strongly disagree, $2=$ disagree, $3=$ neutral, $4=$ agree, $5=$ strongly agree $)$ of all trainees for different aspects of the training experience before and during the COVID-19 pandemic. 


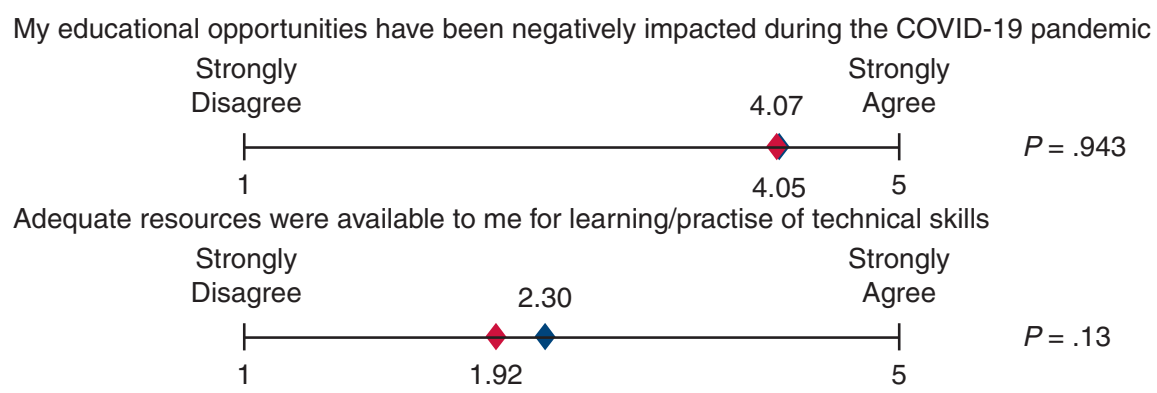

My training was supplemented by the following integrated educational tools: [Webinars]

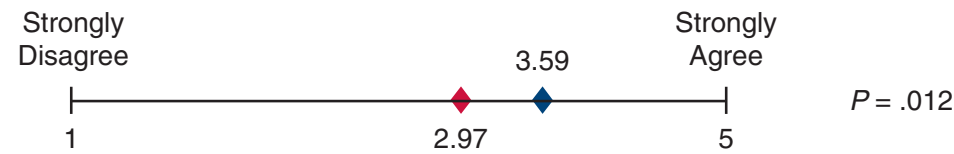

My training was supplemented by the following integrated educational tools: [E-learning]

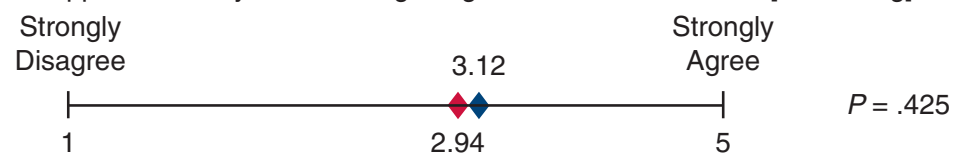

My training was supplemented by the following integrated educational tools: [Surgical simulation training]

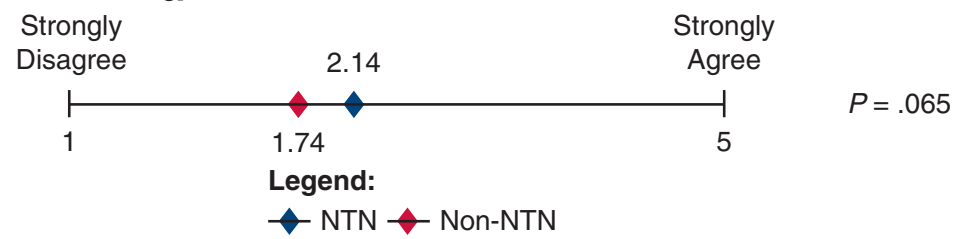

FIGURE 4. Scale comparison using the mean Likert scores $(1=$ strongly disagree, $2=$ disagree, $3=$ neutral, $4=$ agree, $5=$ strongly agree $)$ of all trainees for educational opportunities and resources used during the COVID-19 pandemic. NTN, National Training Number.

Surgical training in the United Kingdom has faced several challenges, including the introduction of reduced working hours to 48 hours per week as a result of the European Working Time Directive, which has been a major crippling factor. ${ }^{10-12}$ In comparison, the weekly working limit in the United States is 80 hours, and advantages of limited working hours other than increased sleep are poorly documented. ${ }^{13}$ In combination with increased patient expectations driving the development of minimally invasive surgery, this adds further complexity in terms of training.

Furthermore, in a 2016 United Kingdom survey study, $86.8 \%$ of trainees reported being willing to spend additional time at work for increased operating opportunities. ${ }^{14}$ This is reflected in our present study, with $60 \%$ of trainees agreeing or strongly agreeing that they attended theatres when not scheduled to gain more operative experience before COVID, which dropped to 19\% during COVID.

In the United Kingdom, cardiothoracic surgical training was recently reduced from an 8-year run-through program to a 7-year program, ${ }^{5}$ with specialization in either cardiac/thoracic specialty selected during the fourth year of training. However, this has been further delayed owing to the disruption caused by COVID- 19 .
During the COVID-19 pandemic, the SCTS Pan London Emergency Cardiac Surgery group advised that only 2 of the 7 cardiac surgical centers in London should provide a cardiac surgical service, a model that was subsequently extended across the United Kingdom. The reduced opportunity for trainees at centers to which services were centralized is overshadowed by the fact that trainees at the remaining centers had less opportunity for training in cardiac surgery, as demonstrated by the drastic reduction in both case volume and trainee-led operations at major cardiac centers. ${ }^{15,16}$ For example, at our institution, 1 of the 2 centers in London providing cardiac surgery as part of the Pan London Emergency Cardiac Surgery protocol, from January 1 to March 20, 2020, there was a total of 430 cardiac operations performed, of which $258(60 \%)$ were performed with consultants as primary operators and $172(40 \%)$ were performed by trainees $(111[64.5 \%]$ by NTN trainees and 61 [35.5\%] by non-NTN trainees). In contrast, only 175 cardiac operations were performed between March 21 and June 7, 2020, of which 101 $(57.7 \%)$ were performed with consultants as primary operators and $74(42.3 \%)$ were performed by trainees (56 [75.7\%] by NTN trainees and 18 [24.3\%] by 


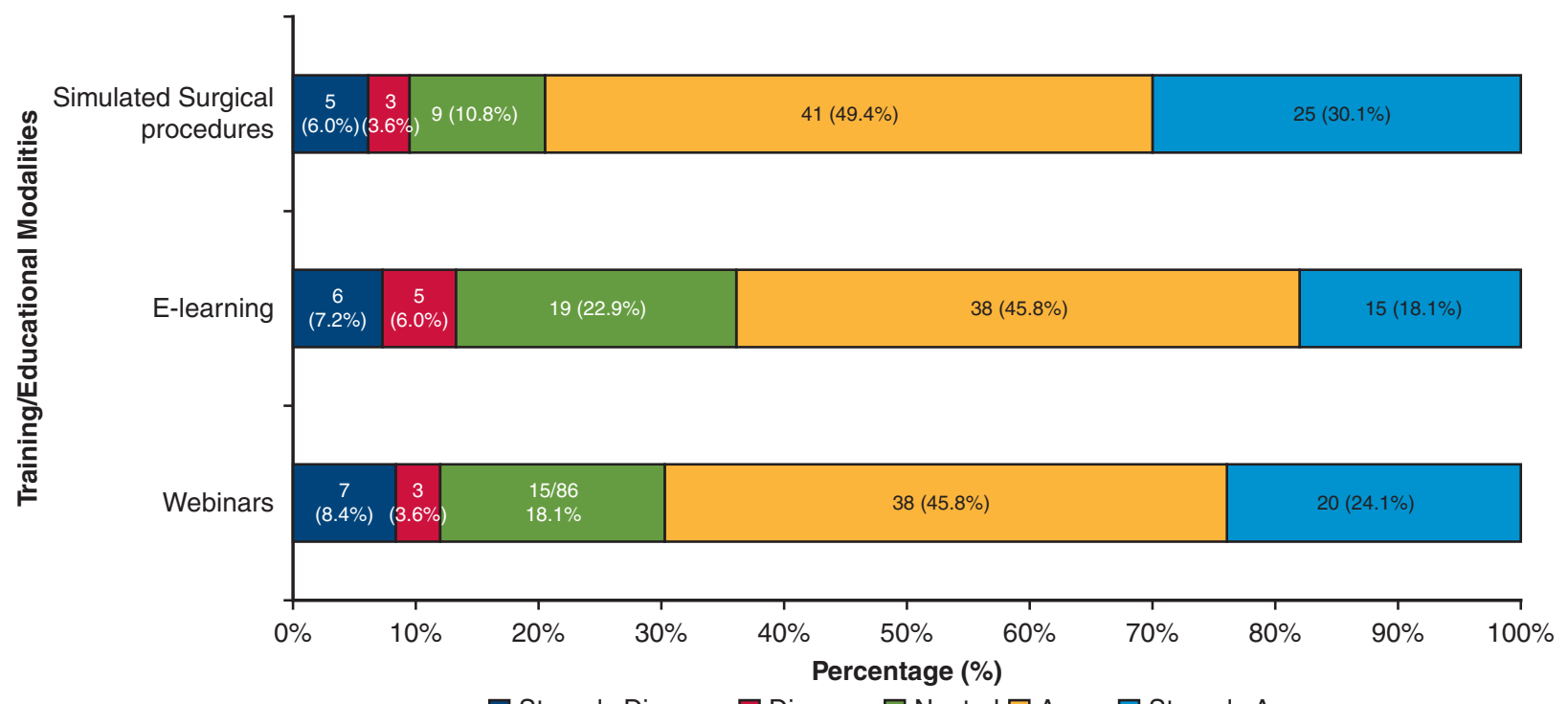

Strongly Disagree $\square$ Disagree $\square$ Neutral $\square$ Agree $\square$ Strongly Agree

FIGURE 5. A $100 \%$ stacked bar graph showing responses on the different modalities that could be used to meet future training/educational training needs, using the Likert scale $(1=$ strongly disagree, $2=$ disagree, $3=$ neutral, $4=$ agree, $5=$ strongly agree $)$. There were a total of 83 responses.

non-NTN trainees). This represents an $59.3 \%$ overall reduction in the number of cardiac operations performed at our institution between the 2 time periods.

In addition, clinical and educational conferences/courses were postponed in an effort to ensure that trainees were available to help healthcare services deal with the pandemic and minimize any potential risk of transmission of the virus. Although this would reduce any potential negative effects on the workforce and subsequently on patients, training was further compromised.

Simulation training should have CPD points accredited

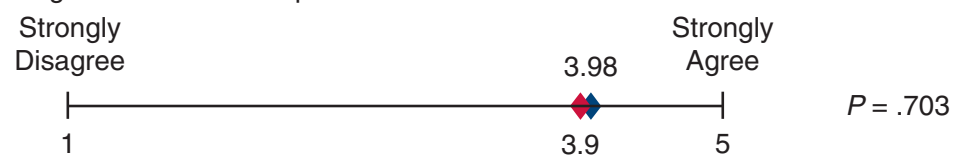

Simulation training is an important tool in improving my surgical skills

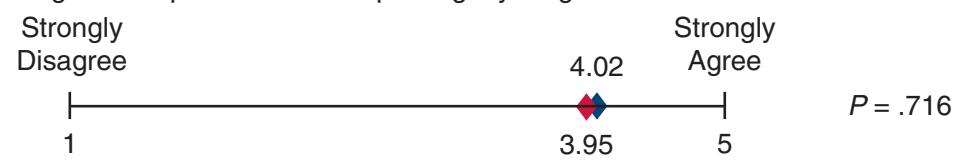

Simulation training has a role in Cardiothoracic Training

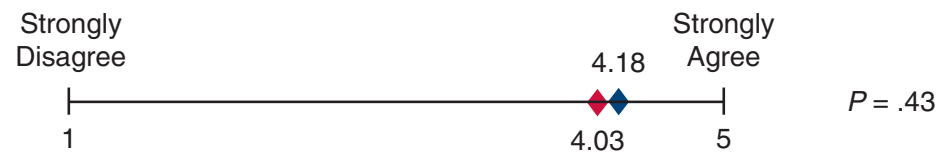

A series of remote wetlab's (where the wetlab kits are sent to you individually in advance, you login online to follow along with the live training and then returning the wet lab kit) would be useful

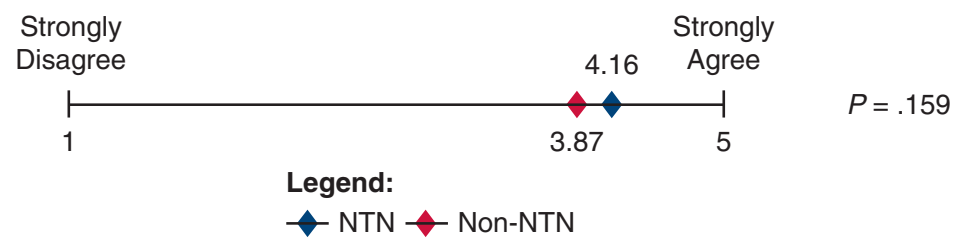

FIGURE 6. Scale comparison using the mean Likert scores $(1=$ strongly disagree, $2=$ disagree, $3=$ neutral, $4=$ agree, $5=$ strongly agree $)$ of National Training Number $(N T N)$ and non-NTN trainees for the use of simulation training in cardiothoracic surgery. CPD, Continuing Professional Development. 


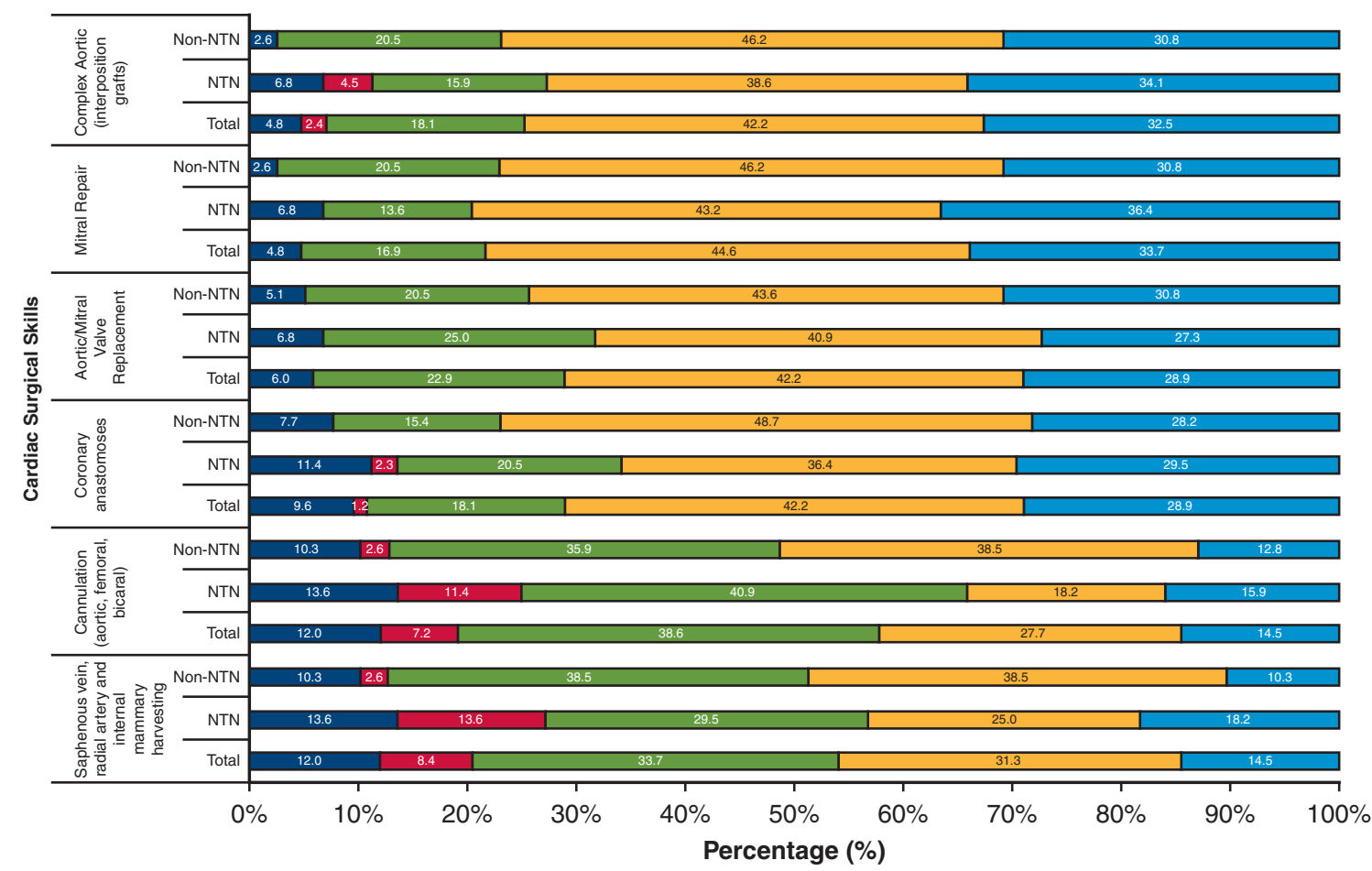

A

$\square$ Strongly Disagree $\square$ Disagree $\square$ Neutral $\square$ Agree $\square$ Strongly Agree

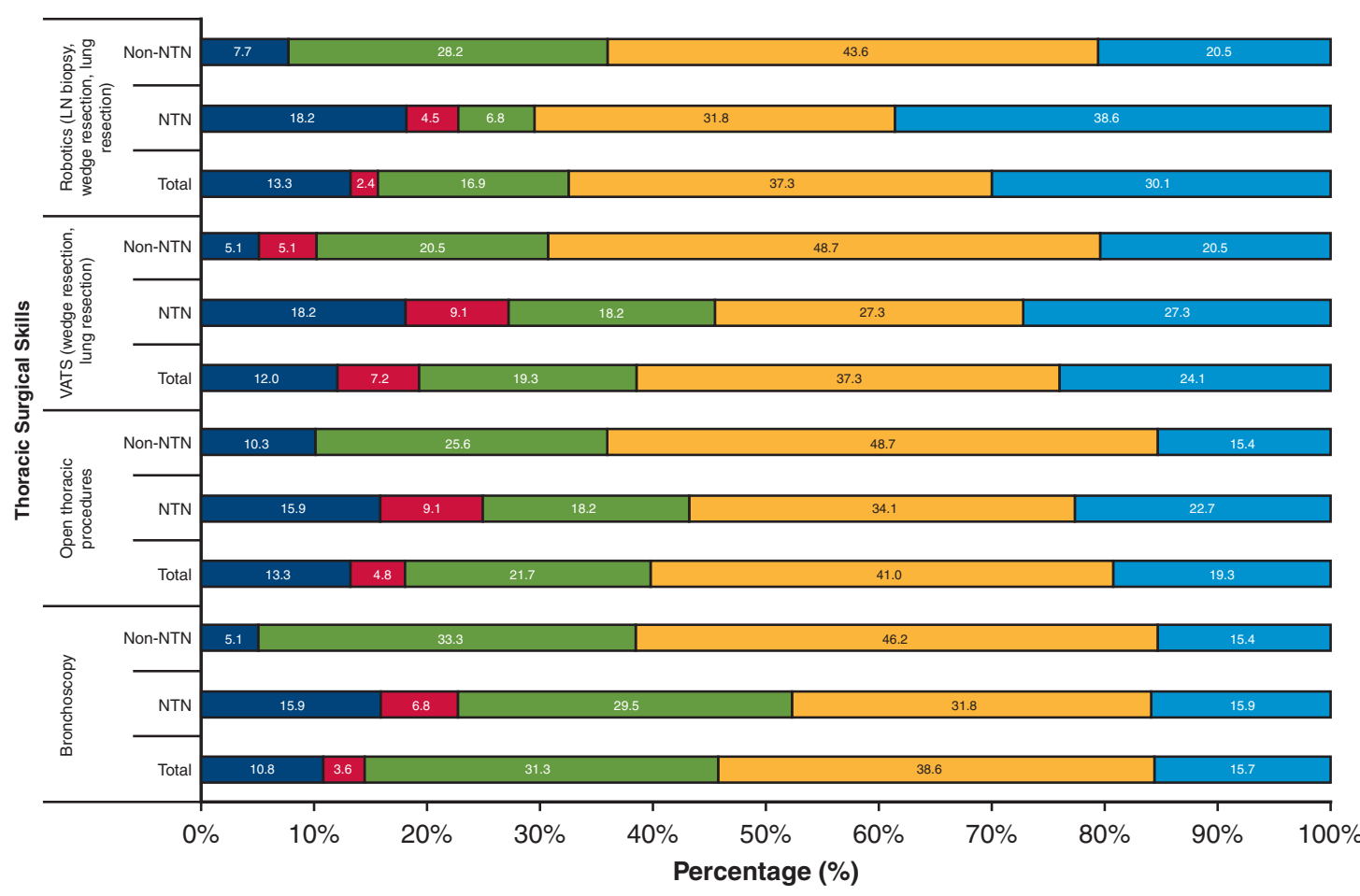

B

Strongly Disagree $\square$ Disagree $\square$ Neutral $\square$ Agree $\square$ Strongly Agree

FIGURE 7. A $100 \%$ stacked bar graph representing different simulation models trainees would find useful in cardiac surgical skills (A) and thoracic surgical skills (B), using the Likert scale $(1=$ strongly disagree, $2=$ disagree, $3=$ neutral, $4=$ agree, $5=$ strongly agree). $C P D$, Continuing Professional Development; NTN, National Training Number; $L N$, lymph node; VATS, video-assisted thoracic surgery. 


\section{Comparison of Cardiothoracic Surgical Training Before and During the COVID Pandemic in the United Kingdom}

\author{
METHODS \\ - Online survey distributed to all UK CTS trainees
}

- Assessing impact of COVID-19 on training and the role of simulation

- Focused on training before and during the COVID-19 pandemic in the UK

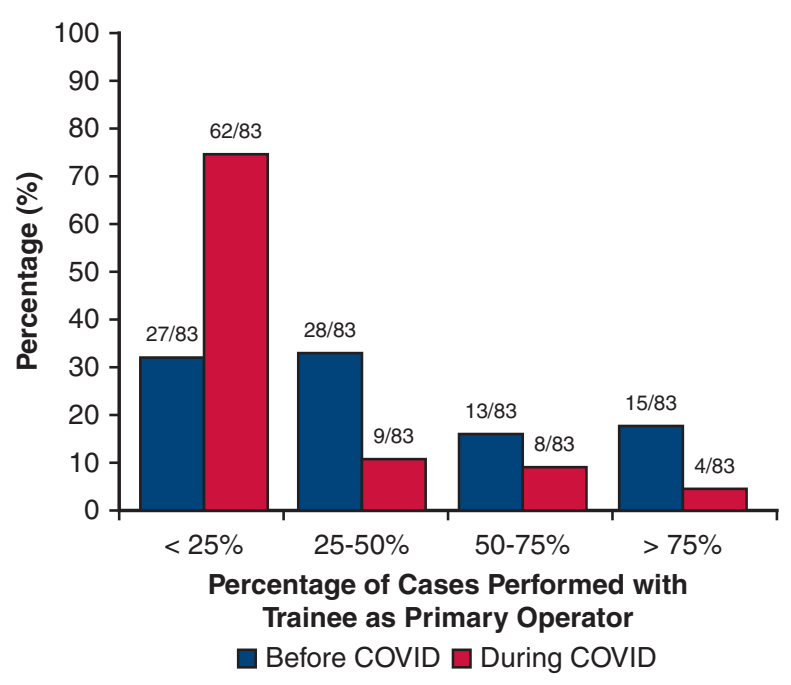

\section{IMPLICATIONS}

- COVID-19 has had a significant impact on Cardiothoracic Surgical Training

- Simulation allows for continued technical skills development outside of the operating room, however wildly underused

Abbreviations: CTS = Cardiothoracic Surgical, NTN = national training number (designated trainee), non-NTN = non-national training number (non designated trainee)

FIGURE 8. Graphical abstract providing an overview of the methods, results, and implication of the study: impact of COVID-19 on training, illustrated by decrease in trainees as primary operators $(P<.001)$. CTS, Cardiothoracic surgery.

In a recent study of national cardiothoracic trainees during the early stages of the lockdown period in the United Kingdom, $55 \%$ of trainees reported a change in their working hours, including longer overall working hours, and a switch to employment in other specialties within the hospital Trust. In addition, $57 \%$ of trainees were redeployed, with the majority required to provide cover in critical care, ${ }^{17}$ similar to the results of our present study (Figure 8).

The impact from multiple lost months of training is substantial. With the ongoing challenges as a result of COVID-19, a number of hurdles with regard to training and accreditation, an important safeguard for the public, must be overcome.

COVID-19 has had a significant impact, with $81 \%$ of trainees agreeing that it has had a negative impact on their training, a finding reiterated in a previous study that found that $90.1 \%$ were somewhat/very concerned about impact on their learning and progression. ${ }^{17}$ The impact can be demonstrated in our study by the number of weekly theatre sessions allocated, which decreased from a mean of 3.1 sessions before the pandemic to 1.7 during the pandemic. This is in addition to the number of cases performed by trainees as the primary operator, as illustrated in Figure 2, and similar to the findings reported by Caruana and colleagues. $^{17}$

Simulation is being used to bridge the gap created by the changes imposed by the European Working Time Directive and patient expectations. It also is an accepted method to increase both basic and more advanced surgical skills in a progressive manner. ${ }^{18}$ In a 2016 survey carried out among UK trainees, $61.5 \%$ of trainees found simulation training useful; however, $62.6 \%$ believed that simulation should not be more prevalent in their training program. ${ }^{14}$

The COVID-19 pandemic has highlighted areas of weakness in the current training structure, and, interestingly, $79.5 \%$ of trainees in our study agreed that simulation should be used to meet the increasing need in training/education moving forward. This is further emphasized by $81 \%$ agreeing that simulation training is an important tool in improving their surgical skills and $87 \%$ agreeing that simulation training has a role in cardiothoracic training. Therefore, it may be important to develop a more extensive compulsory simulation curriculum that is incorporated into the training curriculum, with difficulty adjusted for the level of training. 
The COVID-19 pandemic has had a significant impact not only on training, but also on the well-being of trainees, as demonstrated by the fact that cardiothoracic trainees expressed concerned about their physical $(63 \%)$ and mental $(32 \%)$ well-being. ${ }^{17}$ In addition, ethical dilemmas have surfaced in terms of decision making as a result of the burden on healthcare resources.

\section{Limitations}

There are inherent limitations associated with qualitative studies, and we acknowledge that COVID is an evolving situation in which multiple adaptations have been necessary during the peak of the pandemic. Moreover, data may be subject to researcher bias just as the survey methodology is susceptible to response bias, although responses were anonymized. In addition, as a retrospective study, responses could be affected by recall and perception bias when comparing the 2 time periods. Furthermore, we had a limited response rate $(34.1 \%$ of NTN trainees and $54.9 \%$ of non-NTN trainees), and the results might not be a true representation of the overall demographic. Nonetheless, this survey provides a snapshot of training, with trainee representatives raising a concern that trainees were complaining of survey fatigue because of the number of surveys being sent out during this period in the United Kingdom.

\section{CONCLUSIONS}

The shortcomings in the current surgical training system and challenges faced by trainees have been dramatically highlighted during the COVID-19 pandemic. Simulation has been established to improve trainee development. However, in the United Kingdom, simulation programs are needed to allow continuing practice in the current climate outside of the operating room. Although simulation training may hold the key to improving education and training, it stills needs to be validated with structured programs. The adversities faced during this pandemic can be used as a springboard to advance surgical training and counter the negative impact of the pandemic.

\section{Conflict of Interest Statement}

The authors reported no conflicts of interest.

The Journal policy requires editors and reviewers to disclose conflicts of interest and to decline handling or reviewing manuscripts for which they may have a conflict of interest. The editors and reviewers of this article have no conflicts of interest.

We acknowledge the help and advice of the educational supervisors and trainees who validated the questionnaire, the
SCTS for providing details regarding the number of NTN and non-NTN trainees registered in the United Kingdom, as well as the trainee representatives for helping to distribute the survey.

\section{References}

1. Huang C, Wang Y, Li X, Ren L, Zhao J, Hu Y, et al. Clinical features of patients infected with 2019 novel coronavirus in Wuhan, China. Lancet. 2020;395: 497-506.

2. Senni M. COVID-19 experience in Bergamo, Italy. Eur Heart J. 2020;41: $1783-4$.

3. Bonalumi G, di Mauro M, Garatti A, Barili F, Gerosa G, Parolari A. The COVID-19 outbreak and its impact on hospitals in Italy: the model of cardiac surgery. Eur J Cardiothorac Surg. 2020;57:1025-8.

4. Hassan A, Arora RC, Adams C, Bouchard D, Cook R, Gunning D, et al. Cardiac surgery in Canada during the COVID-19 pandemic: a guidance statement from the Canadian Society of Cardiac Surgeons. Can J Cardiol. 2020;36:952-5.

5. Zakkar M, Benedetto U, Angelini GD, Murphy G, Shah R, Jahangiri M, et al. Cardiothoracic surgery training in the United Kingdom. J Thorac Cardiovasc Surg. 2019;157:1948-55.

6. Joint Committee on Surgical Training. Certification guidelines and checklists; 2020. Available at: https://www.jcst.org/quality-assurance/certification-guide lines-and-checklists/. Accessed December 4, 2020.

7. Joint Committee on Surgical Training. Certification types. Available at: https:// www.jcst.org/introduction-to-training/certification-types/. Accessed December 4, 2020.

8. Al-Jabir A, Kerwan A, Nicola M, Alsafi Z, Khan M, Sohrabi C, et al. Impact of the Coronavirus (COVID-19) pandemic on surgical practice-part 1. Int J Surg. 2020;79:168-79.

9. Ellison EC, Spanknebel K, Stain SC, Shabahang MM, Matthews JB, Debas HT, et al. Impact of the COVID-19 pandemic on surgical training and learner well-being: report of a survey of general surgery and other surgical specialty educators. J Am Coll Surg. 2020;231:613-26.

10. Hurreiz H. The evolution of surgical training in the UK. Adv Med Educ Pract. 2019; 10:163-8.

11. Giles JA. Surgical training and the European working time directive: the role of informal workplace learning. Int J Surg. 2010;8:179-80.

12. West D, Codispoti M, Graham T. The European working time directive and training in cardiothoracic surgery in the United Kingdom. Surgeon. 2007;5: 81-5. quiz 85,121

13. Desai SV, Feldman L, Brown L, Dezube R, Yeh HC, Punjabi N, et al. Effect of the 2011 vs 2003 duty hour regulation-compliant models on sleep duration, trainee education, and continuity of patient care among internal medicine house staff: a randomized trial. JAMA Intern Med. 2013;173: 649-55.

14. Smelt JLC, Phillips S, Hamilton C, Fricker P, Spray D, Nowell JL, et al Simulator teaching of cardiopulmonary bypass complications: a prospective, randomized study. J Surg Educ. 2016;73:1026-31.

15. Abdel Shafi AM, Hewage S, Harky A. The impact of COVID-19 on the provision of cardiac surgical services. J Card Surg. 2020;35:1295-7.

16. Shafi AMA, Atieh AE, Harky A, Sheikh AM, Awad WI. Impact of COVID-19 on cardiac surgical training: our experience in the United Kingdom. J Card Surg. 2020;35:1954-7.

17. Caruana EJ, Patel A, Kendall S, Rathinam S. Impact of coronavirus 2019 (COVID-19) on training and well-being in subspecialty surgery: a national survey of cardiothoracic trainees in the United Kingdom. J Thorac Cardiovasc Surg. 2020;160:980-7.

18. Trehan K, Kemp CD, Yang SC. Simulation in cardiothoracic surgical training: where do we stand? J Thorac Cardiovasc Surg. 2014;147:18-24.e2.

Key Words: surgical training, cardiothoracic surgery, simulation training, surgical education, survey, COVID-19 


\section{APPENDIX 1. SURVEY QUESTIONNAIRE: EFFECT} OF THE COVID-19 PANDEMIC ON

\section{CARDIOTHORACIC SURGICAL TRAINING}

Dear respected colleagues,

We are investigating the impact of COVID-19 on education and training now and the future needs in cardiothoracic surgery. We would be grateful if you could fill out the survey, which will take approximately 8 minutes to complete. Thank you in advance.

*Required

\section{Section 1: Demographics}

1. What is your gender? * Mark only one

- Female

- Male

2. What is your age? *

- Please specify

3. Are you a national trainee $(\mathrm{NTN})$ ? $*$ Mark only one

- Yes

- No

4. If yes, what year of training are you in? Mark only one

- ST1

- ST2

- ST3

- ST4

- ST5

- ST6

- ST7

- ST8

- Post CCT

5. If you are a non-NTN, how many years have you worked as a Senior Fellow in CT surgery in the UK?

- Please specify

6. Are you in full or part-time training? * Mark only one

- Full time

- Part time

7. Which specialty are you in? * Mark only one

- Pure cardiac surgery

- Pure thoracic surgery

- Cardiothoracic

- Major aortic surgery

- Congenital cardiac surgery

- Mechanical support/transplantation

8. Which region are you currently employed? * Mark only one

- London

- East of England

- West Midlands

- East Midlands
- North West

- North East

- Yorkshire and Humber

- South Central

- South East

- South West

- Wales

- Northern Ireland

- Republic of Ireland

- Scotland

\section{Section 2: Service Provision}

9. Hours worked in an average week* Tick all that apply. How many hours did you work in a week, BEFORE COVID, on average?

- Less than 20 hours

- 20 to 36 hours

- 36 to 48 hours

- 48 to 60 hours

- Greater than 60 hours

10. How many extra hours do you work per week, DURING COVID, on average? * Mark only one

- 0

- 1 to 5 hours

- 6 to 10 hours

- 11 to 15 hours

- $>15$ hours

11. Frequency of on-call shifts on a weekday per week $*$ Tick all that apply.

a) How often were you on-call on a weekday, per week, BEFORE COVID?

- None

- One

- Two

- Three or more

b) How often are you on call on a weekday, per week, DURING COVID?

- None

- One

- Two

- Three or more

12. Weekend shifts worked * Tick all that apply.

a) How many weekends did you work in the 2 months preceding COVID?

- None

- One

- Two

- Three

- Four or more 
b) How many weekends have you worked DURING the 2 months of COVID?

- None

- One

- Two

- Three

- Four or more

c) How many weekends did you work in the 2 months preceding COVID?

- None

- One

- Two

- Three

- Four or more

d) How many weekends have you worked DURING the 2 months of COVID?

- None

- One

- Two

- Three

- Four or more

13. Length of weekend shift* Tick all that apply.

a) What was the length of your weekend shift BEFORE COVID?

- 12-hour shift

- 24-hour shift

- 48-hour shift

- I didn't work weekends

b) What is the length of your weekend shift DURING COVID?

- 12-hour shift

- 24-hour shift

- 48-hour shift

- I didn't work weekends

c) What was the length of your weekend shift BEFORE COVID?

- 12-hour shift

- 24-hour shift

- 48-hour shift

- I didn't work weekends

d) What is the length of your weekend shift DURING COVID?

- 12-hour shift

- 24-hour shift

- 48-hour shift

- I didn't work weekends

14. Resident on Call *Tick all that apply.

a) Were you resident on call before COVID?

- Yes

- No b) Were you resident on call during COVID?

- Yes

- No

c) Were you resident on call before COVID?

- Yes

- No

d) Were you resident on call during COVID?

- Yes

- No

15. Job changes during COVID * Tick all that apply.

a) Have you taken on any different roles during COVID?

- Yes

- No

b) Have you been redeployed to other departments?

- Yes

- No

c) Have you taken on any different roles during COVID?

- Yes

- No

d) Have you been redeployed to other departments?

- Yes

- No

16. If you have answered yes in the above question, please specify

\section{Section 3: Surgical Training}

17. Theatre Sessions Allocated (1 session is a morning or an afternoon) * Tick all that apply.

a) How many theatre sessions were allocated to you, per week, BEFORE COVID?

- 1

- 2

- 3

- 4

- 5

- 6

- 7

- 8

- $9+$

b) How many theatre sessions are allocated to you, per week, DURING COVID?

- 1

- 2

- 3

- 4 

- 5
- 6
- 7
- 8
- $9+$

c) How many theatre sessions were allocated to you, per week, BEFORE COVID?
- 1
- 2
- 3
- 4
- 5
- 6
- 7
- 8
- $9+$

d) How many theatre sessions are allocated to you, per week, DURING COVID?
- 1
- 2
- 3
- 4
- 5
- 6
- 7
- 8
- $9+$

18. Primary Operator * Tick all that apply.

a) BEFORE COVID, you were the primary operator in

- $<25 \%$ of cases

- $25 \%-50 \%$ of cases

- $50 \%-75 \%$ of cases

- $>75 \%$ of cases

b) DURING COVID, you are the primary operator in

- $<25 \%$ of cases

- $25 \%-50 \%$ of cases

- $50 \%-75 \%$ of cases

- $>75 \%$ of cases

c) BEFORE COVID, you were the primary operator in

- $<25 \%$ of cases

- $25 \%-50 \%$ of cases

- $50 \%-75 \%$ of cases

- $>75 \%$ of cases

d) DURING COVID, you are the primary operator in

- $<25 \%$ of cases

- $25 \%-50 \%$ of cases

- $50 \%-75 \%$ of cases

- $>75 \%$ of cases
19. Training experience BEFORE COVID * Tick all that apply.

a) The allocated time in theatre was sufficient to gain the required technical skills

- Strongly disagree (1)

- Disagree (2)

- Neutral (3)

- Agree (4)

- Strongly agree (5)

b) I attended theatres when not scheduled to do so to gain more operative experience

- Strongly disagree (1)

- Disagree (2)

- Neutral (3)

- Agree (4)

- Strongly agree (5)

c) My surgical trainer was able to provide me with the training that I required during this period

- Strongly disagree (1)

- Disagree (2)

- Neutral (3)

- Agree (4)

- Strongly agree (5)

d) The allocated time in theatre was sufficient to gain the required technical skills

- Strongly disagree (1)

- Disagree (2)

- Neutral (3)

- Agree (4)

- Strongly agree (5)

e) I attended theatres, when not scheduled to do so, to gain more operative experience

- Strongly disagree (1)

- Disagree (2)

- Neutral (3)

- Agree (4)

- Strongly agree (5)

f) My surgical trainer was able to provide me with the training I required during this period

- Strongly disagree (1)

- Disagree (2)

- Neutral (3)

- Agree (4)

- Strongly agree (5)

20. Training experience DURING COVID * Tick all that apply.

a) The allocated time in theatre was sufficient to gain the required technical skills 
- Strongly disagree (1)

- Disagree (2)

- Neutral (3)

- Agree (4)

- Strongly agree (5)

b) I attend theatres when not scheduled to do so to gain more operative experience

- Strongly disagree (1)

- Disagree (2)

- Neutral (3)

- Agree (4)

- Strongly agree (5)

c) My surgical trainer is able to provide me with the training I require during this period

- Strongly disagree (1)

- Disagree (2)

- Neutral (3)

- Agree (4)

- Strongly agree (5)

d) The allocated time in theatre was sufficient to gain the required technical skills

- Strongly disagree (1)

- Disagree (2)

- Neutral (3)

- Agree (4)

- Strongly agree (5)

e) I attend theatres when not scheduled to do so to gain more operative experience

- Strongly disagree (1)

- Disagree (2)

- Neutral (3)

- Agree (4)

- Strongly agree (5)

f) My surgical trainer is able to provide me with the training I require during this period

- Strongly disagree (1)

- Disagree (2)

- Neutral (3)

- Agree (4)

- Strongly agree (5)

21. Due to the COVID-19 pandemic cardiac surgical services have been centralised across the UK. Has your hospital continued to operate (cardiothoracic procedures) during the COVID-19 pandemic? * Mark only one

- Yes

- No

22. Academia and research * Tick all that apply.

a) Before COVID how many different pieces of research were you involved in during the last year?
- 1

- 2

- 3

- 4

- 5

- 6 or more

b) Before COVID how many presentations to learned societies did you give in the last year?

- 1

- 2

- 3

- 4

- 5

- 6 or more

c) Before COVID how many conferences did you attend in the last year?

- 1

- 2

- 3

- 4

- 5

- 6 or more

d) During COVID, how many different pieces of research have you been involved in?

- 1

- 2

- 3

- 4

- 5

- 6 or more

e) Before COVID, how many different pieces of research were you involved in during the last year?

- 1

- 2

- 3

- 4

- 5

- 6 or more

f) Before COVID, how many presentations to learned societies did you give in the last year?

- 1

- 2

- 3

- 4

- 5

- 6 or more

g) Before COVID, how many conferences did you attend in the last year?

- 1

- 2 

- 3
- 4
- 5
- 6 or more

h) During COVID, how many different pieces of research have you been involved in?
- 1
- 2
- 3
- 4
- 5
- 6 or more

23. Were these education activities funded by:* Mark only one

- Industry

- NHS Trust

- Self-funded

- Combination of two or more from the above

24. To what degree do you disagree/agree with the following statements * Mark only one

a) My educational opportunities have been negatively impacted during the COVID-19 pandemic

- Strongly disagree (1)

- Disagree (2)

- Neutral (3)

- Agree (4)

- Strongly agree (5)

b) Adequate resources were available to me for learning/practise of technical skills

- Strongly disagree (1)

- Disagree (2)

- Neutral (3)

- Agree (4)

- Strongly agree (5)

c) My educational opportunities have been negatively impacted during the COVID-19 pandemic

- Strongly disagree (1)

- Disagree (2)

- Neutral (3)

- Agree (4)

- Strongly agree (5)

d) Adequate resources were available to me for learning/practise of technical skills

- Strongly disagree (1)

- Disagree (2)

- Neutral (3)

- Agree (4)

- Strongly agree (5)

25. My training was supplemented by the following integrated educational tools:* Mark only one a) Webinars

- Strongly disagree (1)

- Disagree (2)

- Neutral (3)

- Agree (4)

- Strongly agree (5)

b) E-learning

- Strongly disagree (1)

- Disagree (2)

- Neutral (3)

- Agree (4)

- Strongly agree (5)

c) Surgical simulation training

- Strongly disagree (1)

- Disagree (2)

- Neutral (3)

- Agree (4)

- Strongly agree (5)

26. Effect of COVID-19 on CCT. Mark only one

a) Do you think you will gain CCT by the planned date?

- Yes

- No

- N/A (post-CCT)

b) Do you think your CCT date should be deferred?

- Yes

- No

- N/A (post CCT)

c) Do you think you will gain CCT by the planned date?

- Yes

- No

- N/A (post CCT)

d) Do you think your CCT date should be deferred?

- Yes

- No

- N/A (post CCT)

\section{Section 4: Now and Future Training/Education}

27. As a consequence of COVID, do you think future training/educational needs should be met by increasing:* Tick all that apply.

a) Webinars

- Strongly disagree (1)

- Disagree (2)

- Neutral (3)

- Agree (4)

- Strongly agree (5)

b) E-learning 
- Strongly disagree (1)

- Disagree (2)

- Neutral (3)

- Agree (4)

- Strongly agree (5)

c) Simulated surgical procedures

- Strongly disagree (1)

- Disagree (2)

- Neutral (3)

- Agree (4)

- Strongly agree (5)

28. If you have an alternative suggestion, please let us know

29. In the year preceding COVID, which of the following simulated surgical training methods have you engaged with? * Mark only one

a) Wetlab (animal/cadaveric tissue)

- Yes

- No

b) Benchtop models

- Yes

- No

c) Virtual reality models

- Yes

- No

d) None

- Yes

- No

30. I would find the following wetlabs useful. * Mark only one per question

a) Saphenous vein, radial artery, and internal mammary artery harvesting

- Strongly disagree (1)

- Disagree (2)

- Neutral (3)

- Agree (4)

- Strongly agree (5)

b) Cannulation (aortic, femoral, bicaval)

- Strongly disagree (1)

- Disagree (2)

- Neutral (3)

- Agree (4)

- Strongly agree (5)

c) Coronary anastomoses

- Strongly disagree (1)

- Disagree (2)

- Neutral (3)

- Agree (4)

- Strongly agree (5) d) Aortic/mitral valve replacement

- Strongly disagree (1)

- Disagree (2)

- Neutral (3)

- Agree (4)

- Strongly agree (5)

e) Mitral repair

- Strongly disagree (1)

- Disagree (2)

- Neutral (3)

- Agree (4)

- Strongly agree (5)

f) Complex aortic (interposition grafts)

- Strongly disagree (1)

- Disagree (2)

- Neutral (3)

- Agree (4)

- Strongly agree (5)

g) Bronchoscopy

- Strongly disagree (1)

- Disagree (2)

- Neutral (3)

- Agree (4)

- Strongly agree (5)

h) Open thoracic procedures

- Strongly disagree (1)

- Disagree (2)

- Neutral (3)

- Agree (4)

- Strongly agree (5)

i) VATS (wedge resection, lung resection)

- Strongly disagree (1)

- Disagree (2)

- Neutral (3)

- Agree (4)

- Strongly agree (5)

j) Robotics (LN biopsy, wedge resection, lung resection)

- Strongly disagree (1)

- Disagree (2)

- Neutral (3)

- Agree (4)

- Strongly agree (5)

31. To what extent do you think this suggestion would improve simulation training? * Tick all that apply.

a) A series of remote wetlabs (where the wetlab kits are sent to you individually in advance, you login online to follow along with the live training and then returning the wet lab kit) would be useful

- Strongly disagree (1)

- Disagree (2) 
- Neutral (3)

- Agree (4)

- Strongly agree (5)

b) Simulation training should have CPD points accredited

- Strongly disagree (1)

- Disagree (2)

- Neutral (3)

- Agree (4)

- Strongly agree (5)

c) Simulation training is an important tool in improving my surgical skills

- Strongly disagree (1)

- Disagree (2)

- Neutral (3)

- Agree (4)

- Strongly agree (5) d) Simulation training has a role in cardiothoracic training

- Strongly disagree (1)

- Disagree (2)

- Neutral (3)

- Agree (4)

- Strongly agree (5)

e) A series of remote wetlabs (where the wetlab kits are sent to you individually in advance, you login online to follow along with the live training and then returning the wet lab kit) would be useful

- Strongly disagree (1)

- Disagree (2)

- Neutral (3)

- Agree (4)

- Strongly agree (5) 